\title{
Efeitos Ambientais sobre Ganho de Peso no Período do Nascimento ao Desmame em Bovinos da Raça Nelore ${ }^{1}$
}

\author{
Claudia Cristina Paro de Paz ${ }^{2,3}$, Lúcia Galvão de Albuquerque ${ }^{3,4}$, Luiz Alberto Fries $3,5,6$
}

RESUMO - O objetivo deste trabalho foi estudar os efeitos de idade da vaca(IDV), idade do bezerro (IDB) e data juliana de nascimento (DJN) sobre o ganho de peso médio diário (GMD) no período do nascimento ao desmame em animais da raça Nelore. Os dados foram analisados por máxima verossimilhança restrita, empregado-se modelo animal. O modelo incluiu os efeitos fixos de grupo contemporâneo (GC), as covariáveis IDV, IDB e DJN e os efeitos aleatórios direto (animal), materno, ambiente permanente e resíduo. As covariáveis foram definidas usando-se polinômios segmentados. Em virtude da alta associação entre os efeitos IDB e DJN e da importância destes efeitos sobre o GMD, optou-se por utilizar estimativas destes dois efeitos em conjunto. O valor da correlação entre IDB e DJN de 0,60 foi considerado indicador de problemas, sendo o gráfico de três dimensões muito útil. O GMD foi significamente influenciado pelos efeitos linear e quadrático da idade de bezerro. Dessa forma, a prática usual de utilizar GMD para obter os pesos ajustados pode não ser adequada. A data juliana de nascimento é um efeito muito importante e, por conseguinte, deve ser estimada preferencialmente à simples inclusão do mês de nascimento no modelo.

Palavras-chave: gado de corte, idade do bezerro, data de nascimento juliana, multicolinearidade

\section{Environmental Effects on Weight Gain from the Birth to Weaning Period in Nellore Breed Cattle}

ABSTRACT - The objective of this work was to study the effects of age of dam (AOD), age of calf (AOC) and Julian date of birth (DOB) on the average daily gain (ADG) from birth to weaning period of Nellore breed animals. The data were analyzed by restricted maximum likelihood, using the animal model. The model included the direct effects of contemporary group (CG), AOD, AOC and DOB, and the direct effects (animal), maternal, permanent environmental and residue. The covariates were defined using segmented polynomials. In view of higher association between the AOC and DOB effects and of the importance of these effects on ADG, estimates with these two effects as a whole were selected. The correlation value between AOC and DOB of -0.60 was a poor indicator of the problems, being the three-dimensional plot very useful. The AGD was significantly influenced by linear and quadratic effects of calf age. The usual way to obtain adjusted weaning weights through AGD can not be adequate for this data set. The julian birth date is an important effect on ADG and should be included in the model and a segmented polynomial function may be preferable to a discrete parameterization.

Key Words: beef cattle, age of calf, Julian date of birth, multicollinearity

\section{Introdução}

A fase de crescimento pré-desmame em gado de corte é considerada muito importante, em virtude das mais altas taxas de crescimento de toda a vida do animal. Durante esta fase são vários os fatores considerados importantes, destacando-se habilidade materna, grupo genético, sexo, mês e ano de nascimento, idade da vaca, idade do bezerro, entre outros.

Para que os animais possam ser comparados e distinguidos na seleção de rebanhos, é necessário que os efeitos de fatores ambientais sobre as características de interesse sejam minimizados. Para isto, podem ser utilizados ajustes e correções para influências ambientais conhecidas (idade do bezerro ao desmame, idade da vaca, data de nascimento dentro do calendário juliano).

No caso de bovinos de corte, os efeitos ambientais importantes para peso ao desmame e ganho de peso no período do nascimento ao desmame que, normalmente, não são incluídos no grupo contemporâneo e podem ser pré-ajustados são idade do bezerro ao desmame, idade da vaca e data de nascimento no calendáriojuliano.

A Beef Improvement Federation (BIF) (1996)

${ }^{1}$ Parte da Dissertação apresentada à FCAV-UNESP pelo primeiro autor como parte das exigências para a obtenção do título de Mestre em Zootecnia. Área de concentração: Genética e Melhoramento Animal. Financiada pelo CNPq.

2 Pesquisador Científico - Instituto de Zootecnia.

${ }^{3}$ Departamento de Zootecnia - Melhoramento Genético Animal- FCAV - UNESP - Jaboticabal, SP.

${ }^{4}$ Pesquisador do CNPq.

${ }^{5}$ GenSys Consultores Associados S/C Ltda.

${ }^{6}$ FAPESP. 
recomenda que sejam feitos ajustes do peso do bezerro ao desmame (PD) para idade do bezerro, como efeito linear, e idade da vaca (fatores aditivos), considerado como efeito quadrático simples (Polinômio Ordinário). Quanto à data juliana de nascimento, não há menção.

O efeito da idade da vaca (IDV) é conseqüência principalmente da habilidade materna, mais especificamente da capacidade de produção de leite no decorrer de sua vida útil. Em geral, o peso dos bezerros à desmama aumenta com IDV, até esta atingir a maturidade fisiológica, época em que as vacas mostram maior desempenho nas características de habilidade materna. Após a maturidade, a tendência é de redução no peso dos bezerros.

A maioria dos trabalhos em que se estudou o efeito da idade da vaca ao parto sobre as características de crescimento no período pré-desmame utilizou, como opção de modelagem, os Polinômios Ordinários, observando a existência de efeito quadrático. No entanto, alguns autores têm mostrado a existência de efeito Quadrático-Quadrático (Polinômio Segmentado). FRIES (1984), em animais da raça Hereford, CAMPOS et al. (1989) e LUCERO et al. (1995), em animais da raça Nelore, estudaram IDV sobre o ganho de peso do nascimento ao desmame (GMD), utilizando Polinômios Segmentados. Esses autores modelaram o efeito de IDV sobre GMD, de acordo com Polinômio Quadrático-Quadrático. CAMPOS et al. (1989) encontraram, para o GMD, diferenças de até $7 \%$ nos fatores de correção (multiplicativos) para IDV em machos e de até $6 \%$ em fêmeas.

A idade do bezerro ao desmame (IDB) é fator de grande influência sobre o ganho de peso no período do nascimento ao desmame. Diversos trabalhos indicam que o ajuste linear padrão (205 dias de idade) para IDB não remove toda a variação resultante deste efeito no GMD, mesmo dentro de intervalo de 90 dias, mostrando efeito não-linear de IDB sobre o GMD (FRIES, 1984; AHUNU e MAKARECHIAN, 1986 e 1987; WOODWARD et al., 1989; e WOLF, 1996).

Na maioria dos trabalhos zootécnicos, quando se leva em consideração o efeito da data de nascimento (DJN), este é incluído no modelo de análise pela simples inclusão do mês de nascimento. No entanto, recentemente, alguns autores têm recomendado o cálculo de fatores de correção para este efeito, preferencialmente à simples inclusão do mês de nascimento no modelo de análise. FRIES (1984), com animais da raça Hereford criados no Rio Grande do Sul, e CAMPOS et al. (1989), com animais da raça Nelore, observaram melhor desempenho dos animais nascidos cedo - julho/agosto (dentro da Primavera). Assim, quanto mais tarde nasce o animal desconsiderando-se que ele será desmamado mais novo e, por conseguinte, o efeito é contrário e de maior magnitude - pior é seu desenvolvimento. Já na produção de outono, espera-se o inverso, pois os animais nascidos no fim do verão estão mais predispostos às doenças peri-natais e encontram baixa qualidade forrageira quando iniciam sua alimentação (ao redor dos 5 meses) por intermédio de pastejo. FRIES (1996b) observou efeito da data de nascimento sobre o peso à desmama. A maior concentração de nascimentos ocorreu na época mais favorável do ano ( $2^{\mathrm{o}}$ semestre), mostrando a existência de efeito bioeconômico importante da data de nascimento sobre os pesos à desmama. Entre os extremos, a diferença foi superior a $20 \mathrm{~kg}$.

Os objetivos do presente trabalho foram estudar os efeitos IDV, IDB e DJN sobre o GMD de bovinos da raça Nelore e discutir as dificuldades de se estimarem os efeitos IDB e DJN.

\section{Material e Métodos}

Os 18.772 registros analisados no presente trabalho foram obtidos junto à GenSys Consultores Associados S/C Ltda., com a autorização do seu proprietário (Granja Rezende S.A.). Foram analisados os GMD de animais da raça Nelore, nascidos na estação da primavera, no período de 1984 a 1994.

A edição dos dados foi efetuada de forma a se obter conjunto estruturado que permitisse a realização das análises necessárias para a estimação de parâmetros genéticos e a determinação de fatores de correção para o ganho médio diário de peso no período compreendido entre o nascimento e o desmame. Dessa forma, as informações que pudessem interferir nas análises foram eliminadas. A edição dos dados foi efetuada de acordo com os seguintes passos:

1. Criação das variáveis, IDB (em dias - em classes de 10 em 10 dias, variando de 15 a 30); IDV (em anos - classes de 1 em 1 ano, variando de 4 a 15); DJN (em dias - em classes de 20 em 20 dias, variando de 10 a 18); o ganho de peso do nascimento ao desmame nãoajustado (GND); GMD e DJN. Assim, foram criadas IDB, IDV e DJN, como variáveis classificatórias, para efetuar os testes dos ajustes prévios e verificar o comportamento das funções do ganho de peso prédesmame, em função de IDB, IDV e DJN, para posterior modelagem pelos Polinômios Segmentados e Ordinários, conforme será explicado a seguir. 
2. Criação da variável grupo contemporâneo pela concatenação das variáveis sexo, fazenda, retiro, manejo, data juliana de desmame e ano de nascimento do bezerro.

3. Eliminação dos animais nascidos no outono, em virtude do pequeno número de animais nascidos nesta estação. Os animais cruzados também foram eliminados, restando apenas os animais puros $\mathrm{PO}$ e PC. O arquivo ficou então com 25.731 registros.

4. Eliminação dos animais cujas mães tinham menos de 4 anos e mais de 15 anos. A eliminação das novilhas (mães com três anos de idade) ocorreu devido às diferenças existentes nas práticas de manejo realizadas na fazenda, exclusivamente com estes animais, sem que estas diferenças estivessem codificadas no arquivo, portanto, não podendo ser detectadas, o que poderia alterar os resultados. Os animais cujas mães tinham mais de 15 anos foram eliminados simplesmente pelo pequeno número de observações; restaram então 19.820 registros.

5. Avaliação do número de filhos por touro, eliminando-se os animais, cujos touros tinham menos de quatro filhos, resultando em 19.754 registros.

6. Avaliação do número de observações por grupo contemporâneo, eliminando-se os grupos contemporâneos com menos de quatro animais, resultando em 18.784 registros.

7. Reavaliação do número de filhos por touro, eliminando-se os touros com menos de quatro filhos, resultando em 18.772 animais, filhos de 7619 vacas e 109 touros.

8. Novamente o arquivo foi submetido à contagem do número de observações por grupo contemporâneo e não houve alteração do número de registros.

Durante a edição dos dados, foram testadas as pré-correções dos registros de ganho total de peso pré-desmame que vêm sendo efetuadas pela Granja Rezende S.A. (fatores de correção obtidos por CAMPOS et al., 1989), para os efeitos de IDV, IDB e DJN, com o objetivo de verificar se os dados estão sendo bem ajustados.

Para efetuar este teste, foi realizada estimação dos efeitos da idade da vaca, idade do bezerro ao desmame e data juliana de nascimento sobre o ganho em peso prédesmame ajustado(GNDA), pelas análises de variância, utilizando-se o método dos quadrados mínimos, por intermédio do procedimento GLM(SAS, 1992), utilizando-se modelo com os seguintes efeitos fixos: grupo contemporâneo, idade da vaca, idade do bezerro ao desmame e data juliana de nascimento.

Depois de testar as pré-correções, foram estudadas as funções polinomiais na tentativa de modelar as curvas dos efeitos IDV, IDB e DJN dentro da estação, sobre o GMD. Estas funções estão relacionadas abaixo.

\section{Funções testadas}

São descritas a seguir as funções polinomiais (Polinômios Segmentados e Polinômios Ordinários) testadas neste trabalho, para os três efeitos estudados (IDV, IDB e DJN):

1. Para a idade da vaca:

- Regressão Quadrática Simples (Polinômio Ordinário)

$\mathrm{Y}_{\mathrm{X}}=\mathrm{a}_{0}+\mathrm{a}_{1} \mathrm{X}+\mathrm{a}_{2} \mathrm{X}^{2}$

- Polinômio Segmentado Quadrático-Quadrático

$\mathrm{Y}_{\mathrm{X}}=\mathrm{a}_{0}+\mathrm{a}_{1} \mathrm{X}+\mathrm{a}_{2} \mathrm{X}^{2}+\left(\mathrm{b}_{2}-\mathrm{a}_{2}\right) \mathrm{Z}$

em que $Z=0$, se $X<K$; $\mathrm{Z}=(\mathrm{X}-\mathrm{K})^{2}$, se $\mathrm{X}>\mathrm{K}$.

2. Para a Idade do Bezerro do Desmame:

- Polinômio Segmentado Quadrático-Linear

$\mathrm{Y}_{\mathrm{X}}=\mathrm{a}_{0}+\mathrm{a}_{1} \mathrm{X}+\mathrm{b}_{2} \mathrm{Z}$

em que $Z=0$, se $X>K$; $Z=(X-K)^{2}$, se $X<K$.

- Polinômio Segmentado Quadrático-Quadrático (Conforme 2)

- Regressão Quadrática Simples (Polinômio Ordinário)

(Conforme 1)

3. Para a Data de Nascimento:

- Polinômio Segmentado Quadrático-Quadrático (Conforme 2)

- Regressão Linear Simples (Polinômio Ordinário) $\mathrm{Y}_{\mathrm{X}}=\mathrm{a}_{0}+\mathrm{a}_{1} \mathrm{X}$;

Nos modelos acima descritos, YX é GMD; X é IDV, ou IDB, ou DJN, respectivamente, para cada efeito; $a_{0}, a_{1}, a_{2}$ e $b_{2}$ são os parâmetros de cada função; e K é o "nó" ou ponto de junção entre os segmentos dos Polinômios Segmentados.

\section{Modelagem dos efeitos ambientais}

Para a modelagem dos efeitos de ambiente, foram seguidas as seguintes etapas: (1) os efeitos IDV, IDB e DJN foram incluídos em modelo fixo, como variáveis discretas (para IDB e DJN foram utilizadas classes de 10 e 20 dias, respectivamente) juntamente com GC; (2) por inspeção dos diagramas de pontos das soluções obtidas, diferentes polinômios segmentados foram testados e os nós foram determinados com base nos quadrados médios dos resíduos; (3) o modelo final foi determinado por ponderação de testes estatísticos, magnitude dos efeitos e capacidade de explicação biológica.

(1) Modelagem dos polinômios segmentados:

Depois de testada a significância dos efeitos de idade da vaca, idade do bezerro ao desmame e data juliana de nascimento, sobre o ganho de peso do 
nascimento ao desmame ajustado (GNDA), determinaram-se o comportamento e a forma da curva para cada um destes três efeitos, sobre o ganho médio diário de peso não-ajustado (GMD), utilizando-se o método dos quadrados mínimos, por intermédio do procedimento GLM (SAS, 1992), usando um modelo com os seguintes efeitos fixos: grupo contemporâneo, idade da vaca, idade do bezerro ao desmame, data de nascimento do bezerro, touro e vaca.

Observou-se que todos os efeitos foram altamente significativos, no entanto, a forma da função ao longo do domínio de $\mathrm{X}$ (variável independente-efeito da idade da vaca) não permitiu explicação biológica para o efeito da idade da vaca. $\mathrm{O}$ ganho de peso médio diário aumentou linearmente com o aumento da idade da vaca.

Para os demais efeitos - idade do bezerro e data juliana de nascimento - as formas de ambas as funções ao longo do domínio de $\mathrm{X}$ possibilitaram explicação biológica. $\mathrm{O}$ ganho de peso médio diário diminuíu linearmente com o aumento da idade do bezerro e apresentou comportamento quadrático para a data juliana de nascimento, com aumento do ganho médio diário até a $12^{\mathrm{a}}$ classe de data juliana de nascimento (dias 240 -259), na qual atingiu seu máximo, diminuindo até a $18^{\text {a }}$ classe de data juliana de nascimento (dias 360 -365).

Então, repetiu-se esta mesma análise, retirandose do modelo o efeito fixo de touro e não foi observada nenhuma alteração no comportamento de cada uma das curvas.

Resolveu-se, então, testar o mesmo modelo com única alteração, que foi a retirada do efeito fixo de vaca (efeito absorvido) do modelo. Todos os efeitos ambientais (grupo contemporâneo, idade da vaca, idade do bezerro e data juliana de nascimento) foram altamente significativos, como em todos os outros modelos descritos anteriormente, com única diferença, o comportamento da função ao longo do domínio de X (variável independente - efeito da idade da vaca) possibilitou explicação biológica para o efeito da idade da vaca.

Deve-se ressaltar, com relação à opção ABSORB do SAS, que, ao se efetuar este procedimento no presente conjunto de dados absorvendo vacas, observou-se que, provavelmente, devido ao grande número de dados (18772 animais filhos de 7619 vacas), ocorreram interferências da opção ABSORB nos resultados, não permitindo que as análises fossem confiáveis (PAZ, 1997).

Em seguida, os dados foram analisados utilizando-se um modelo animal, empregando-se o programa computacional MTDFREML (BOLDMAN etal. 1993).

O modelo incluiu os efeitos fixos de grupo contemporâneo, idade da vaca (separada para machos e fêmeas), idade do bezerro ao desmame e data juliana de nascimento como variáveis classificatórias e os efeitos aleatórios de animal (efeito genético aditivo direto), vaca (efeito genético aditivo materno), ambiente permanente (associado à vaca) e resíduo.

De acordo com a literatura, o efeito da idade da vaca ao parto de machos e fêmeas é estudado separadamente, assumindo-se a existência de interação entre estes dois efeitos (IDV e sexo). A Granja Rezende S.A. já utiliza fatores de correção separados (para machos e fêmeas) para este efeito.

Esta análise, empregando o programa computacional MTDFREML, em que os efeitos ambientais pré-ajustáveis foram colocados no modelo como variáveis classificatórias, foi realizada apenas para comparar estes resultados com os resultados encontrados na análise realizada pelo procedimento GLM (SAS, 1992). As curvas encontradas para os efeitos de idade do bezerro, data juliana de nascimento e idade da vaca, empregando-se modelo animal, foram muito próximas às curvas encontradas utilizando-se o procedimento GLM (SAS, 1992), no modelo sem o efeito da vaca. Esta última análise confirmou a possível interferência da opção ABSORB do SAS, nos resultados, conforme citado anteriormente.

A definição dos polinômios deu-se após a modelagem das curvas dos efeitos em estudo (IDV, IDB e DJN) sobre o ganho médio diário de peso pré-desmame (GMD).

Definidos os Polinômios Segmentados (FULLER, 1969), ou Polinômios Ordinários a serem utilizados, foram criados os conjuntos de covariáveis.

A idade da vaca foi modelada como efeito Quadrático-Quadrático, com o ponto de junção ("nó") aos oito anos de idade (para machos e fêmeas). A idade do bezerro foi descrita por regressão quadrática simples (Polinômio Ordinário). A data de nascimento dentro do calendário juliano foi descrita por meio de regressão linear simples (Polinômio Ordinário).

(2) e (3) Estimação do "nó" ou ponto de junção

O maior obstáculo para o uso de Polinômios Segmentados é que os "nós" ou pontos de junção devem ser conhecidos. O conhecimento prévio na área técnica específica, o uso de diagramas e médias para a estimação grosseira dos "nós" ou o uso de modelo não-linear para a estimação simultânea dos "nós" e dos coeficientes de regressão podem ser empregados nesta tarefa.

GALLANT e FULLER (1973) propuseram ver- 
são generalizada do método de GAUSS-NEWTON, modificado por HARTLEY, para resolver o problema (não-linearidade) de encontrar estimativas dos coeficientes de regressão em cada segmento da curva e da posição dos "nós" que minimizaria a soma de quadrados dos resíduos. No caso de uma curva de forma quadrática-quadrática, como a utilizada no presente estudo para o efeito da idade da vaca sobre o ganho de peso médio do nascimento à desmama, a convergência é garantida. Para encontrar os valores iniciais para o processo iterativo, esses autores recomendam obter diagramas de pontos e escolher valores iniciais por inspeção. Usando estes valores iniciais para os "nós", podese obter os valores iniciais para os outros parâmetros por métodos comuns de regressão múltipla.

No presente trabalho, foram escolhidos os possíveis "nós" das curvas por meio da inspeção visual dos pontos em gráfico de dispersão. Esse procedimento auxilia a visualização do possível número de segmentos que compõem a função, além dos prováveis pontos em que ocorrem mudanças na curvatura. O próximo passo foi alterar o valor do nó iterativamente, de maneira a se encontrar o ponto em que a soma de quadrados do resíduo (SQR) fosse minimizada. Depois de detectado o "nó" estimado que indicou ponto crítico de transição das fases, procedeu-se à estimação dos parâmetros do modelo. Este método é aparentemente trabalhoso, no entanto, é exeqüível e bastante prático, em decorrência da facilidade de computação dos polinômios.

Oúnico efeito modelado como polinômio segmentado foi o da idade da vaca, que foi modelado como Polinômio Segmentado Quadrático-Quadrático, que é uma função composta por dois segmentos quadráticos e quatro parâmetros livres. Os parâmetros $\mathrm{a}_{0}, \mathrm{a}_{1} \mathrm{e} \mathrm{a}_{2}$ indicam, respectivamente, o intercepto, o coeficiente de regressão linear e o coeficiente de regressão quadrático, do primeiro segmento polinomial. $\mathrm{O}$ parâmetro $\left(b_{2}-a_{2}\right)$ expressa a diferença de quadratura entre o primeiro e o segundo segmento quadrático e a soma de $\left(a_{2}+\left(b_{2}-a_{2}\right)\right)$, que é igual $a b_{2}$ determina a curvatura do segundo segmento quadrático. O "nó" ou ponto de junção foi estimado aos 8 anos de idade da vaca.

As análises foram realizadas para o ganho de peso médio diário no período do nascimento ao desmame (GMD), em que foram geradas total de 58.243 equações de modelos mistos. A idade da vaca foi considerada em anos e a idade do bezerro e a data juliana de nascimento, em dias. O número de níveis para o efeito de grupo contemporâneo foi de 613; o número de vacas, 7619; o número de touros, 109; o número de animais que tiveram seus desempenhos medidos, 18.772; o número de animais na matriz de parentesco, 25.001; e o número de covariáveis, 9 .

$\mathrm{Na}$ forma matricial, o modelo linear utilizado pode ser descrito como:

em que

$$
\mathrm{Y}=\mathrm{X} \beta+\mathrm{Za}+\mathrm{Mm}+\mathrm{Wp}+\mathrm{e}
$$

$\mathrm{Y}=$ Vetor da variável dependente (GMD);

$\mathrm{X}=$ Matriz de incidência de efeitos fixos;

$\beta=$ Vetor dos efeitos fixos (grupo contemporâneo, como variável classificatória e as covariáveis idade da vaca ao parto e do bezerro ao desmame e data juliana de nascimento);

$Z=$ Matriz de incidência de efeitos genéticos aditivos diretos;

$\mathrm{a}=$ Vetor de valores genéticos aditivos diretos dos animais;

$M=$ Matriz de incidência dos efeitos genéticos aditivos maternos;

$\mathrm{m}=$ Vetor de valores genéticos aditivos maternos;

$\mathrm{W}=$ Matriz de incidência do efeito de ambiente permanente da vaca;

$\mathrm{p}=$ Vetor de efeito de ambiente permanente da vaca; e $\mathrm{e}=$ Vetor de efeitos residuais aleatórios.

$$
E=\left[\begin{array}{c}
\beta \\
a \\
m \\
p \\
e
\end{array}\right]=\left[\begin{array}{l}
\beta \\
0 \\
0 \\
0 \\
0
\end{array}\right] ; \quad \operatorname{Var}\left[\begin{array}{l}
a \\
m \\
p \\
e
\end{array}\right]=\left[\begin{array}{cccc}
\sigma_{a}^{2} A & \sigma_{a m} A & 0 & 0 \\
\sigma_{a m} A & \sigma_{m}^{2} A & 0 & 0 \\
0 & 0 & \sigma_{p}^{2} I_{C} & 0 \\
0 & 0 & 0 & \sigma_{e}^{2} I_{N}
\end{array}\right]
$$

* A covariância entre os efeitos genéticos direto e materno $\left(\sigma_{\mathrm{am}}\right)$ foi considerada como sendo igual a zero.

\section{Resultados e Discussão}

\section{Efeito da idade da vaca}

$O$ efeito da idade da vaca dentro do sexo do bezerro foi modelado como Polinômio Segmentado Quadrático-Quadrático, (FULLER 1969), com "nó" aos oito anos de idade. Os bezerros (machos) filhos de vacas jovens sofreram maior restrição alimentar (menor produção de leite das vacas jovens) que as bezerras (fêmeas), no período pré-desmame. O GMD aumentou dos 4 aos 6 anos de idade da vaca, quando atingiu ganho máximo, diminuindo depois até os 15 anos. Resultados semelhantes foram encontrados com animais da raça Hereford (FRIES, 1984) e em outros estudos realizados no Brasil Central (CAMPOS et al., 1989; LUCERO et al., 1995; e FRIES, 1996a).

\section{Utilização dos polinômios segmentados}

O uso de polinômios segmentados é uma técnica não 
muito difundida. Normalmente, utilizam-se, para o ajustes de curvas, os Polinômios Ordinários (polinômios comumente utilizados nas análises de regressões).

Os Polinômios Ordinários são aqueles que aproximam uma função média por meio de única função de relação entre as variáveis explanatórias (independentes) e a resposta (variável dependente). Estes polinômios apresentam vários problemas para a análise estatística, como a multicolinearidade, a desuniformidade na determinação da função ao longo do domínio de X (variável independente) e a dependência do comportamento da função na área de maior concentração de pontos (SCHENKEL, 1989).

Nos estudos realizados na área zootécnica, são comumente usados os Polinômios Ordinários de primeira e de segunda ordem e funções não-lineares. Os polinômios de graus mais elevados são de difícil interpretação biológica e as funções não-lineares exigem processos iterativos muito complexos para a estimação de seus parâmetros e também apresentam parâmetros de difícil interpretação biológica.

RICE (1969) demonstrou que Polinômios Ordinários são inadequados em muitas situações, especialmente quando se trabalha com funções e dados do mundo biológico. As funções que expressam as relações biológicas são, em geral, de natureza dissociada, ou seja, o comportamento das variáveis em uma região da curva pode ser completamente independente do comportamento delas em outras regiões dos possíveis valores, sendo, dessa forma, melhor definidas pelos Polinômios Segmentados. Os Polinômios Segmentados ou funções "Splines" que foram definidos por RICE (1969) como "segmentos de polinômios de grau $\mathrm{P}$, conectados em pontos denominados de "nós", de forma a terem derivadas contínuas de grau P-1", são alternativa para solucionar este problema. Os "nós" ou pontos de junção indicam o ponto onde a curva muda o comportamento (inclinação ou grau do polinômio).

Segundo FULLER (1969), uma função deve ser: (1) contínua; (2) apresentar primeiras derivadas contínuas; (3) ser de fácil estimação (linear nos parâmetros); (4) permitir com facilidade a computação de alguns critérios qualitativos e propriedades dos estimadores.

Os Polinômios Segmentados são funções contínuas, possuem primeiras derivadas contínuas, mas são nãolineares nos seus parâmetros e, portanto, estimáveis por processos iterativos. $\mathrm{Na}$ determinação dos segmentos que compõem a função, primeiramente utiliza-se o diagrama de pontos, com objetivo de detectar, por inspeção, os pontos de mudança de comportamento da curva.

A dificuldade no uso de Polinômios Segmentados está na determinação dos "nós" ou pontos de junção entre os segmentos.

Efeito da idade do bezerro ao desmame

O ganho de peso médio diário apresentou média geral observada de $0,681 \mathrm{~kg} / \mathrm{dia}$ e variou dentro do intervalo de 156 a 308 dias (IDB). Esse ganho decresceu à medida que aumentou a idade do animal, sendo que a queda foi mais acentuada entre 156 e 200 dias; deste ponto, até 308 dias, a taxa de ganho de peso apresentou declínio suave.

Em princípio, a idade do bezerro foi definida como Polinômio Segmentado Quadrático-Linear, com ponto de junção entre estes dois segmentos ("Nó") estimado aos 180 dias. Esta definição baseou-se no diagrama de pontos, por inspeção, e na contínua experimentação para encontrar o ponto cuja soma de quadrados do resíduo (SQR) fosse minimizada. No entanto, quando se definiu, no modelo completo (todos os efeitos, ambientais e genéticos), o efeito da idade do bezerro ao desmame como Polinômio Segmentado Quadrático-Linear ou como Polinômio Quadrático Ordinário, a soma de quadrados do resíduo (SQR) e o $\mathrm{R}^{2}$ $(0,771192)$ mantiveram-se constantes.

Esta característica (GMD), geralmente, é estudada separadamente do efeito da idade bezerro. A Beef Improvement Federation (BIF) recomenda usar pesos e ganhos de pesos ajustados linearmente para a idade do bezerro (períodos de 90 dias).

Os resultados obtidos neste estudo mostram a existência de "super-ajustamento" na média dos bezerros mais jovens e "sub-ajustamento" na média dos bezerros mais velhos, quando é feito o ajustamento linear da idade do bezerro sobre o GMD. Este efeito sozinho pode dificultar a avaliação genética dos touros de Inseminação Artificial com relação aos touros do próprio rebanho ou touros de repasse.

Efeitos não-lineares de IDB sobre características de crescimento em bovinos de corte foram observados por FRIES (1984), CAMPOS et al. (1989), LUCERO et al. (1995) e FRIES (1996b).

Se no lugar da variável GMD fosse utilizada a variável peso ao desmame (PD), provavelmente o grau do polinômio usado para descrever esta relação precisaria ser de maior grau. $O$ efeito cúbico sobre o PD tem o mesmo significado que o quadrático sobre GMD. Talvez a discussão dos resultados fosse mais fácil com $\mathrm{PD}$, mas o problema da multicolinearidade entre as covariáveis IDB e DJN seria aumentado.

Efeito da data juliana de nascimento

À semelhança do efeito de idade do bezerro ao 
desmame, a data de nascimento no calendário juliano, em princípio, foi modelada como Polinômio Segmentado Quadrático-Quadrático, que, como foi descrito anteriormente, éfunção composta por dois segmentos quadráticos, com o ponto de junção ("Nó") estimado em 300 dias.

Esta função foi escolhida com base no diagrama de pontos por inspeção e por meio de testes contínuos para encontrar o ponto cuja soma de quadrados do resíduo (SQR) fosse minimizada.

No entanto, quando se incluiu no modelo completo (todos os efeitos ambientais e genéticos) este efeito como polinômio quadrático-quadrático, ocorreram problemas com o efeito da idade do bezerro houve inversão total - ou seja o ganho médio diário do bezerro mudou de sentido e passou a aumentar com o aumento da idade do bezerro.

No Brasil Central há grande concentração de nascimentos durante a estação da seca, que é o período mais favorável. Neste estudo, os nascimentos ocorreram entre os dias 220 e 365 do calendário juliano. O coeficiente de regressão linear negativo estimado indica que os nascimentos ocorridos mais cedo na estação são mais vantajosos. Estes resultados estão de acordo com os resultados dos trabalhos mencionados anteriormente, mas todos têm chegado a descrições mais elaboradas deste efeito.

Junção dos efeitos de idade do bezerro com a data de nascimento

Os efeitos da data juliana de nascimento e da idade do bezerro ao desmame foram difíceis de modelar. Os "nós" e as funções polinomiais usados para modelar estes efeitos foram dependentes e/ou influenciados pela modelagem utilizada para os outros efeitos, sugerindo problemas de multicolinearidade e/ou concentração de dados em pontos influentes, além de influenciar as funções que determinam os outros efeitos sobre o GMD.

Este fato pode ser atribuído ao alto valor encontrado para a correlação entre os resíduos das covariáveis idade do bezerro ao desmame e data juliana de nascimento, depois de removido o efeito do grupo contemporâneo. Com a incorporação da data juliana de desmame na definição de grupo contemporâneo, criou-se correlação perfeita entre IDB e DJN para os animais nascidos em cada grupo contemporâneo. O valor encontrado para este coeficiente de correlação amostral entre os resíduos destes efeitos foi de $-1,00$ com $\mathrm{P}<0,001$, indicando problemas de multicolinearidade. O coeficiente de correlação entre estas duas covariáveis foi de -0,60. Este alto valor da correlação entre os resíduos destas duas covariáveis não causou surpresa, já que estavam sendo encontrados problemas na modelagem destas duas covariáveis, mostrando estreita relação entre a idade do bezerro ao desmame e a data juliana de nascimento. Dessa forma, estas duas covariáveis não puderam ser tratadas independentemente; por isso, decidiu-se pelo cálculo de fatores de correção para os efeitos da idade do bezerro ao desmame e da data juliana de nascimento sobre o ganho médio diário de peso prédesmame, com base na distribuição conjunta destas covariáveis (PAZ, 1997).

HOFFMANN e VIEIRA (1977) explicam os casos de ortogonalidade e multicolinearidade que podem ocorrer na matriz X, quando se trabalha com regressão linear múltipla. Quando a Matriz X é ortogonal, então $X^{\prime} X$ é matriz diagonal e, portanto, as estimativas dos parâmetros da regressão múltipla coincidem com as estimativas dos coeficientes das regressões lineares simples de Y com cada uma das variáveis independentes. Nos casos que ocorre a multicolinearidade perfeita, a matriz X possui colunas linearmente dependentes, ou seja, regressão linear múltipla com duas variáveis "independentes" perfeitamente correlacionadas entre si. Segundo esses autores, as principais consequências da multicolinearidade são: "1) as variâncias e covariâncias das estimativas serão muito elevadas, isto é, as estimativas obtidas podem ter erros muito grandes, que podem estar altamente correlacionados entre si. A baixa precisão das estimativas torna difícil, sendo impossível distinguir as influências das diversas variáveis independentes; 2 ) pode-se eliminar variáveis da análise, porque os coeficientes não se mostraram estatisticamente diferentes de zero e na realidade estas variáveis podem ser importantes e a amostra disponível é que não permite detectar sua influência; e 3) as estimativas dos coeficientes variam bastante de amostra para amostra. A adição de algumas observações à amostra pode alterar muito o valor da estimativa obtida.

Assim, foram verificadas as idades do bezerro existentes e, para cada idade, as correspondentes faixas de datas de nascimento no calendário juliano, para então efetuar o cálculo dos fatores de correção para estes dois efeitos.

Desta forma, com base no princípio da parcimônia, e procurando-se reduzir a correlação múltipla entre as colunas de $\mathrm{X}$, pela redução do número de colunas de X, optou-se por modelar o efeito da idade do bezerro ao desmame, como regressão quadrática (Polinômio Ordinário), e o efeito da data juliana de 
nascimento como regressão linear simples.

Estes resultados estão em concordância com os resultados de FRIES (1984), WOODWARD et al. (1989) e WOLF (1996), que mostraram a não linearidade do efeito da idade do bezerro ao desmame sobre o ganho de peso pré-desmame, e mais ainda sobre o peso à desmama.

O número de observações para o ganho médio diário de peso pré-desmame, em função da idade do bezerro e da data juliana de nascimento, está representado na Tabela 1 e Figura 1.

A correlação de $-0,60$ entre IDB e DJN e a distribuição das frequências observada na Tabela 1 não mostram o quanto os dados estão mal distribuídos nas datas de nascimento e idades ao desmame, dando a falsa impressão de que há boa distribuição das freqüências, e que assim, estes efeitos poderiam ser estimados.

Pela análise da Figura 1, nota-se que os nascimentos não estão distribuídos ao longo do ano todo. Desta forma, informações que são obtidas com a Tabela 1 são nulas quando comparadas com as informações da Figura 1. Pode-se observar células vazias vs células ocupadas na Tabela 1, mas é difícil visualizar, algumas vezes, a magnitude dos números em cada uma das células. A Figura 1 mostra claramente que há margem muito pequena para estimar estes dois efeitos. O nível de associação entre os dois efeitos pode ser observado na Figura 1. A forma destes efeitos físicos e os problemas causados pela multicolinearidade (estimativas dos erros-padrão altamente inflacionadas e problemas numéricos na obtenção de estimativas razoáveis) ajudam entender porque é tão difícil estimar estes dois efeitos conjuntamente.

Os efeitos físicos também foram negativamente associados e os bezerros nascidos mais cedo na estação foram favorecidos em relação à DJN, podendo ter sofrido interferências, já que estes animais são desmamados mais tarde. Para os bezerros nascidos mais tarde na estação, a recíproca é verdadeira. Estes resultados estão de acordo com os trabalhos citados anteriormente (FRIES, 1984; CAMPOS et al., 1989; LUCERO et al., 1995; e FRIES, 1996b).

Assim, os efeitos da idade do bezerro e da data de nascimento foram tratados conjuntamente. Ao se verificar cada uma das idades dos bezerros, para cada faixa de data de nascimento no calendário juliano, observou-se que os dados apresentaram-se concentrados em determinados pontos e que alguns pontos não apresentaram algum dado (Tabela 1 e Figura 1).

A concentração dos nascimentos e das idades do bezerro encontra-se entre os dias 270 e 350 da data juliana de nascimento ou seja, no período de 27 de setembro a 16 de dezembro, no calendário gregoriano, e entre 230 e 290 dias de idade do bezerro, como pode ser observado na Figura 1, pela distribuição das freqüências para o ganho médio diário de peso prédesmame em função da idade do bezerro e da data juliana de nascimento.

A tabela de distribuição das freqüências apresentada juntamente com a Figura 1 não permite a visualização da grande concentração dos dados, mas o gráfico das freqüências dos números observados mostra mais claramente esta concentração.

A concentração dos dados em pontos influentes pode ter agravado ainda mais a interferência da multicolineridade entre os efeitos da idade do bezerro e da data juliana de nascimento.

Estes resultados estão em concordância com a distribuição encontrada por FRIES (1996b), que trabalhando com 730.578 animais zebuínos cadastrados na ABCZ mostrou a ocorrência de maior concentração de nascimentos no segundo semestre do ano (entre os dias 210 e 300).

A ocorrência da maior concentração de nascimentos, que pode ser observada no período de setembro a dezembro e de 230 a 290 dias idade (Figura 1), não coincide com o período em que os animais apresentaram maior taxa de ganho de peso, que foi observado no intervalo de 220 a 270 do calendário juliano ( 8 de agosto a 27 de setembro no calendário gregoriano) e no inter-

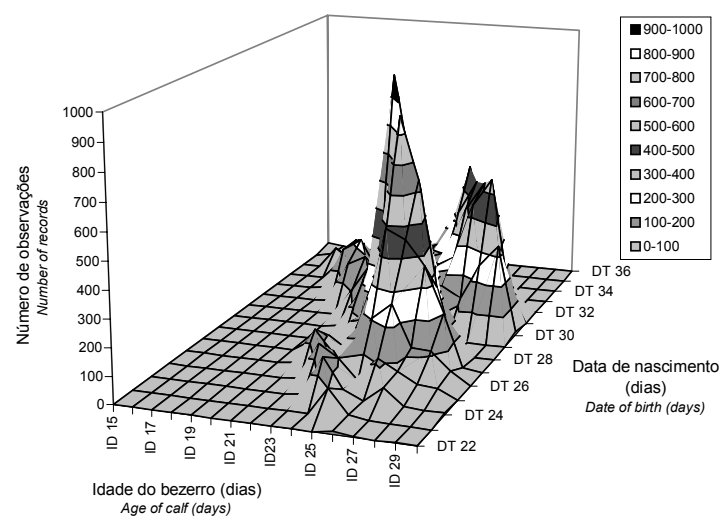

Figura 1- Número de observações de acordo com a idade do bezerro ao desmame (ID) e com a data juliana de nascimento (DT).

Figure 1- Number of records according to age of calf (AC) and date of birth (DB). 
valo de 210 a 250 dias de idade do bezerro (PAZ, 1997).

Desta forma, realizando-se melhor controle dos nascimentos dos animais - animais nascendo mais cedo na estação da primavera (entre 220 e 270 dias no calendário juliano, que representa de 9 de agosto a 27 de setembro no calendário gregoriano) - e desmamando-se animais com menores idades (em torno de 210 e 250 dias), pode-se obter incremento no sistema de produção de carne. Poder-se-ia calcular quanto a produtividade deste rebanho mudaria, se sua população nascesse entre os dias 220 e 270 (agostosetembro).

Com base na distribuição dos dados, em relação a estes dois efeitos, estimou-se o ganho médio diário de peso pré-desmame para cada faixa de idade do bezerro conjuntamente com cada faixa de data de nascimento no calendário juliano. A extrapolação, ou estimação dos fatores de correção para idade do bezerro, independentemente da data de nascimento no calendário juliano, apresentou valores absurdos.

O acúmulo de maior número de dados, procurando melhorar a distribuição destes dados em relação à idade do bezerro ao desmame e a data juliana de nascimento, pode amenizar a interferência da multicolinearidade.

Considerando que a data de nascimento influencia o ganho médio diário de peso pré-desmame de maneira relevante, pode-se supor que o efeito sobre a produção de leite da vaca e sobre suas reservas corporais também seja expressivo. Dessa forma, em sistemas com parições durante todo o ano, o intervalo de partos médio pode estar sendo alongado desnecessariamente, com repercussão econômica. Este problema pode ser reduzido com o estabelecimento de estação de monta adequada.

Como recomendação, a prática mundial de ajuste do peso à desmama, ou funções deste, apenas para os efeitos linear e quadrático da idade da vaca e linear da idade do bezerro, deve ser verificada para cada conjunto de dados com volume, qualidade e importância suficientes.

\section{Conclusões}

O uso de GMD para pesos corrigidos para a idade desconsidera importantes componentes de idade.A idade do bezerro ao desmame apresentou efeitos linear e quadrático sobre GMD. A data juliana de nascimento é efeito muito importante e, portanto, deve ser estimado, preferencialmente, usando registros de diversos anos e rebanhos e várias estações reprodutivas. Fatores de correção conjuntos para os 
efeitos IDB e DJN podem ser estimados e usados quando a associação entre estes efeitos for muito intensa, sendo estes fatores preferíveis à simples inclusão do mês de nascimento no modelo de análise.

\section{Agradecimentos}

Ao Dr. Antonio Batista Sancevero e à Granja Rezende S.A., pelo fornecimento dos dados, e ao Dr. Vanerlei Mozaquatro Roso, pela pré-edição dos dados.

\section{Referências Bibliográficas}

AHUNU, B., MAKARECHIAN, M. , 1986. Influence of birth date, sex of calf, breed group and age of dam on preweaning performance of range beef calves. Can. J. Anim. Sci., 66(2):381-388.

AHUNU, B., MAKARECHIAN, M. Preweaning patterns of growth in three breed groups of range beef calves. Can. J. Anim. Sci., 67(3):653-661, 1987.

BIF. Guidelines for uniform beef improvement programs. Raleigh: U. S. Dept. Agriculture - North Carolina State University. 1996, $155 \mathrm{p}$.

BOLDMAN, K.G., KRIESE, L.A., VAN VLECK, L.D. et al., 1993. A manual for use of MTDFREML. A Set of Programs to Obtain Estimates of Variances and Covariances. USDAARS, Clay Center, 120p.

CAMPOS, L.T., SILVA, P.R., FRIES, L.A. 1989. Fatores de correção para efeitos ambientais que afetam o ganho do nascimento à desmama em bovinos da raça Nelore. Coletânia de Pesquisas Inéditas sobre Zebu. Empresa de Pesquisas Agropecuária de Minas Gerais. EPAMIG. Uberaba, MG. p.108-123.

FRIES, L. A. 1984. A study of weaning weights in Hereford cattle in the state of Rio Grande do Sul Brazil. Ph.D. Thesis. Iowa State University, Ames, Iowa. p. 317.

FRIES, L. A, Efeito da idade da vaca sobre o peso à desmama em zebuínos. In: SIMPÓSIO NACIONAL DE MELHORAMENTO ANIMAL, 1, 1996, Ribeirão Preto. Anais ... Ribeirão Preto: SBMA, 1996a. p.255-256.

FRIES, L. A, Efeito da data de nascimento sobre o peso à desmama em zebuínos. In: SIMPÓSIO NACIONAL DE MELHORAMENTO ANIMAL, 1, 1996, Ribeirão Preto. Anais... Ribeirão Preto: SBMA, 1996b. p.249-250.

FULLER, W.A. 1969. Grafted polynomials as approximating fuctions. Austr. J. Agric. Econ., 13:35-46.
GALlANT, A. R., FULLER, W. A. 1973. Fitting Segmented Polynomial Regression models whose join points have tobe estimated. J. Anim. Stat. Assoc., 68:140-147.

HOFFMANN, R., VIEIRA, S. 1977. Regressão linear múltipla. In: HOFFMANN, R., VIEIRA, S. Análise de Regressão. São Paulo: HUCITEC-EDUSP. p.107-166.

LUCERO, C.E., CAMPOS, L.T., CARDOSO, F.F. et al. Fatores de correção do ganho de peso pré-demama, pela idade da mãe e do bezerro, e data de nascimento, na raça Nelore. In: REUNIÃO ANUAL DA SOCIEDADE BRASILEIRA DE ZOOTECNIA, 32, 1995, Brasília, DF. Anais.... Brasília: SBZ, 1995. p.616-618.

PAZ, C.C.P. Efeitos ambientais e genéticos que afetam o ganho de peso pré-desmame em bovinos da raça Nelore. UNESP, Jaboticabal, SP, 1997. 117 p. Dissertação (Mestrado em Ciências Veterinárias) - Universidade Estadual de São Paulo, 1997.

PEIXOTO, A. M. 1993. Fatores que interferem no crescimento do gado de corte até a desmama. In: Bovinocultura de Corte. Fundamentos da Exploração Racional.p.129-158. Piracicaba: FEALQ (550p).

RICE, J. R. The approximation of functions. V. II. AddisonWeslay, Massachusetts. 1969.

SAS User's Guide: Statistical Analysis Systems. Cary, 1992.

SCHENKEL, F. S. Utilização de Polinômios Segmentados na Pesquisa Zootécnica: Considerações Teóricas e Práticas, Porto Alegre: Universidade Federal do Rio Grande do Sul, 1989. 51p. (Notas).

WOODWARD, B. W., POLLAK, E. J., QUAAS, R. L. 1989. Adjusting weaning weights of Simmental beef calves to an age-constant basis. J. Anim. Sci., 67(1):20-27.

WOLF, P. G. L. Efeitos da heterose individual e materna sobre o ganho de peso do nascimento ao desmame de terneiros Pampiano-Braford. Porto Alegre, 1996. 114p. Dissertação (Mestrado em Ciências Veterinárias) - Faculdade de Veterinária, Universidade Federal do Rio Grande do Sul, 1996.
Recebido em: $13 / 03 / 98$

Aceito em: 10/08/98 